\title{
Vertexes in kinetic space-matter with local stresses instead of localized particles with distant gravitation
}

\author{
Igor É. Bulyzhenkov \\ Moscow Institute of Physics and Technology, Institutsky lane 9, Dolgoprudny, Moscow reg., 141700, Russia \\ Lebedev Physics Institute RAS, 53 Leninsky pros., Moscow, 119991, Russia \\ bulyzhenkov.ie@mipt.ru
}

\begin{abstract}
Due to the fact that negative energies have no existence in physical reality, the advanced mechanics of purely positive energies should describe gravitational interactions and collisions in monistic terms of extended kinetic energies and their local stresses. Such non-Newtonian mechanics of continuous inertial densities reinforces the Cartesian concept of matter-extension in the metric formalism of Einstein-Grossmann with a supplemental (dark, aether) fraction of bi-vertex mass-energy distributions. Local accelerations or decelerations of mono-vertex material densities in a multi-vertex distribution of complete kinetic energy arise under its constant integral due to nonlocal organization of continuous densities. Such integral conservation of the distributed mass-energy occurs instantaneously throughout the whole continuum of correlated densities and metric stresses despite the time-varying contributions of complementary monovertex and bi-vertex fractions. Under the nonlocal organization of purely kinetic (positive) mass-energy, geodesic self-heating and self-cooling of the pulsating space-matter conserve the integral energy in the two-fraction virial theorem for the averaged motion of visible mono-vertexes in the presence of invisible bi-vertex (interference, dark) mass-energy. Metric stresses of such material space are subordinate to nonlocal self-government of continuously distributed kinetic energy, including the relativistic rest-energy of General Relativity. These mutually consistent or correlated stresses in inertial space-time-energy create timelessly coordinated self-accelerations, observed for dense material volumes as distant gravitational pulls. In order to falsify/verify the nonlocal self-organization of adaptive kinetic energy, the monistic mechanics of self-consistent inertial densities and metric stresses can suggest moderate field changes in the temporal redshift, cycles of geodetic falls and takeoffs in pulsating kinetic organizations, and the calculated acceleration of the expanding Metagalaxy in its current phase of geodesic self-cooling.

Keywords: Nonlocal mass-energy, Pulsating self-organization, Metric monism, Geodesic

cooling/warming, Continuous mass, Bi-vertex energy
\end{abstract}

Preprint submitted to Particle

February 18, 2021 


\section{Introduction to matter-extension}

The compelling evidence for accelerating 'dark energy' in cosmology got many scientific laboratories of multiple countries involved in the search for invisible material entities. The ongoing search for dark entities is based on the conventional dogma that Newtonian mechanics will forever remain for the low speed dynamics of inertial masses. Only slight modifications of Newton's empty space scheme for inertia and gravity might be assumed 'in the area of theory applications'. Newtonian approximations were indeed well tested in practice for the slow motion of mechanical bodies in the fields of the Earth and the Sun. There are no mathematical reasons to deny the classical mechanics of point masses as the successful model of terrestrial events and many cosmic measurements (accepting for a moment that negative gravitational energies also belong to the physical world of observations). Today it is not physicists and mathematicians, but philosophers who are still calling to find the principle errors of Newton: "Einstein's theory can be accepted only with the recognition that Newton's was wrong" [1. But will modern mechanics with tests on super-colliders be able to hear the quiet skepticism of philosophers?

Physicists are currently not taking into account sophisticated Eastern philosophies. But even after a superficial acquaintance with the Yin-Yang dialectic, it seems logical to associate Newton's negative potential (and the negative gravitational self-energy, $-m c^{2}$, derived below) with the incorporeal (yin) world, and not with the physical reality of positive kinetic energies. To develop better thermal physics of the corporeal (yang) world, it is necessary to associate metric fields with partial losses of positive kinetic energies or with corresponding shifts in inside rest-energies $m c^{2}$ of Einstein. Again, the spatial distribution of only inertial (positive, themokinetic) energies can form the physical reality and observable values for measurements. Nobody measured the negative gravitational energy of Newton despite some people attempting to quantize it in formal calculations. Metricized densities of quantized matter in terms of continuous mechanical fields were indeed expected by Einstein from General Relativity (GR) rather than the formal geometrization of Newtonian gravitational pulls [2]. Astronomers can only measure the coordinate velocity of distant bodies, not their physical velocity in the local metric. And one can analyze that the Einstein-Grossmann geodesic fall of the probe body in extreme fields increases the physical velocity of this body $d x^{i} / \sqrt{g_{o o}} d t \rightarrow c$ only, and decreases the coordinate velocity of real spatial displacements $d x^{i} / d t \rightarrow 0$. Einstein's revolutionary dynamics does contradict the Newton monotonous fall to the field center and predicts kinetic pulsations of falling-bouncing matter in strong metric fields 3. 
By accepting quantum entanglement in macroscopic measurements and spooky nonlocality in cosmos [4, 5, it is time to also infer that presumable separated inertial masses and gravitational fields in the Newton paradigm of empty space might not correspond at all to the physical reality. The latter might be better approximated, in principle, by Cartesian matter-extension [6, 7]. In short, classical gravity frivolously associated the forced motion of inertial bodies with the 1686 invention of negative potentials. But negative, unmeasurable energies are witnesses of pseudoscience models in reasonable interpretations of mechanical events.

If Thomas Kuhn was right in 1962, then theoretical proves of Newtonian fundamental fallacies are as relevant today as the experimental search for dark energy. There are no kinetic tensions in the empty (massless) space, only 'the divine action-at-a-distance' can be postulated by such a model of mechanical states with the negative gravitational energy. According to the author's understanding of Eastern thinkers and Russian cosmists, negative energies have no existence whatsoever in the physical reality of observations. Contrary to Newton, Einstein assigned only positive (kinetic) energies $\gamma m c^{2} \sqrt{g_{o o}}>0$ to all mechanical bodies in all metric fields. Below we revise the misleading empty (and curved) space and reject pseudo-scientific negative energies of (also non-existing) point masses from the proposed metric thermomechanics of distributed kinetic densities in nonlocal self-organization of continuous mass-energy. We employ the Cartesian matter-extension with competing mono-vertex and bi-vertex energies on their inside and shared degrees of freedom for inside relativistic chaos and observable kinetic order, respectively. Bi-vertex inertial densities of space-matter will represent the lost (dark) fraction of thermal (kinetic) energy in our thermomechanics, which is in alternative view to the Newton theory of localized masses in empty space without its own temperature. We find that for such monistic mechanics of kinetic energy that metricized nonlocal distribution of mass-energy can generate the pushing self-tension for the GR local self-acceleration $c^{2} u^{\nu} \nabla_{\nu} u_{\mu}$ of inertial densities, where $c u^{\mu} \equiv c d x^{\mu} / \sqrt{g_{\rho \lambda} d x^{\rho} d x^{\lambda}}$ is their relativistic four-velocity. In other words, nonlocal metric theory of two-fraction kinetic energy can coherently remove unphysical point masses and needless negative energies from Einstein's scientific heritage.

Einstein often mentioned the urgent need to understand the structure of total energy, which he called in German 'gesamtenergie'. It might formally have few topological parts for kinetic densities and the negative self-potential $\left(-m c^{2}\right)$ next to the positive kinetic self-energy $\left(+m c^{2}\right)$ of Special Relativity (SR). Then, the pro-Newtonian world of zero-balanced kinetic and potential energies could be formally extended on the yin-yang composition of relativistic partners in line with Chinese dialectical thinking. In accordance with this thinking, a self-contained metric generalization of positive inertial densities should avoid such a Newtonian trap with the formal mixture of corporeal and incorporeal energies in classical 
mechanics. We have to recall several metaphysical reasons prior to arriving at the novel quantitative analysis of monistic mechanics for only positive kinetic energies. Here positive thermokinetic densities can arrange local self-pushes within the correlated metric continuum of nonlocal mass-energy in order to understand the local kinetic reason of the 'divine action at-a-distance'.

Aristotle's space-plenum was material and self-governed [8]. All bodies in such material space have powers that collectively explain the existing ordering in the organization of the mechanical world of inertial bodies. The concept of void space regions without matter was also unclear to Descartes $[6,7]$, who considered the continuous extension of matter or 'matter-extension' as the non-empty space plenum. This material plenum was logically considered in the Aristotle's Metaphysics after Plato said in the Timaeus that "matter and space are the same". Newton's 'successful' dynamics of point-like masses dropped for a while the need for Cartesian matter-extension with sophisticated grids of vortexes in 1644 "Philosophiae Naturalis". Even nowadays, Newton's "Philosophiae Naturalis Principia Mathematica" for localized masses and continuous gravitation forces in empty space still dominates for the low speed references of relativistic dynamics. Not Aristotle's space-plenum or Cartesian material space, but Newtonian empty space modeling became mandatory for 1916 Einstein's tensor gravitation 9] in the limit of weak metric fields. Staying with the monistic Cartesian views, the author tends to relate the further development of Einstein's GR with replacement of its external references (from the Newton gravity) with the SR self-references for thermokinetic energy. Classical mechanics failed to find the negative gravitational selfenergy of the point mass because of the integral divergence $\int m \delta(\mathbf{x})(-G m /|\mathbf{x}|) d^{3} x \rightarrow-\infty$. Nonetheless, textbook physics of point masses with the positive rest-energy is still mixing yin and yang worlds in one theory for observable bodies. But Chinese dialectics cannot accept for the natural Universe such a mystical, pseudo-scientific gathering of both measurable (inertial, yang) and unmeasurable (gravitational, yin) energies in the self-consistent description of only measurable events. Some radical steps are required to revise or reject Newtonian gravitation and its present penetration into Einstein's geometrization of energy in order to remove the current conflict of yin and yang notions in the modeling of thermokinetic reality of the nonlocal Universe.

Let us compare once more the main propositions of Newton and Descartes. Newton argued since 1686 that the coordinate space is immovable and non-material, that material bodies are localized objects moving relative to the coordinate space (and to each other), that the point inertial particle is the source of long-range forces, that intangible coordinate forces continuously fill the entire immovable space (the dualism of fields and sources was formulated later, after Faraday's studies), that inertial masses are constant under the spatial motion, and that there are no equations for the state of the elementary point 
mass. Descartes stated in 1644 and earlier that each particle or mechanical body is a non-uniform vortex of time-varying moving densities, there is no emptiness in reality, that only circular motions of material densities are stable, that all bodies are expanded entities and their vortex structures are in the pushing contact directly with each other, that there must be equations for the extended elementary object, and that there should be a parametrization (referred to today as geometrization) of the Universe's material space.

Being originally designed for measurable events, pro-Newtonian gravimechanics (and electrodynamics) of retarded fields relies only on superficial experimental observations and mathematical models for observed phenomena. The pragmatic motto 'Nullius in verba' has indeed been very beneficial to begin the technical progress in Europe after the Middle Ages. The Newtonian world of localized inertial bodies had such tremendous quantitative successes that the Chinese reading of the natural Universe through alternative worlds (of measurable and immeasurable contributors) was ignored for a while by mechanics. However, 'Book of Changes', the foundation for Chinese mentality and culture, has distinguished the parallel complementarity of two (corporeal, measurable and incorporeal, immeasurable) worlds and not their mix. Should physics ignore the Eastern philosophical treasury just to satify all initial and palliative models? Why should physicists follow the incorporeal (yin) gravitational pseudo-energy in empty space, say in the current search for dark energy? How can it be proved that General Relativity can selfconsistently comply only with the corporeal (yang) side of dialectically paired worlds? We plan to address some of these fundamental questions in a way consistent with the Einstein metric theory of thermokinetic energies. GR can be self-refereed by its SR dynamics instead of the negative (yin, fiction) potential from the unrealistic model of the point mass.

The metric approach to thermokinetic material densities will maintain the cyclic dynamics of inside chaos and kinematic order of inertial mass-energy. Its adaptive self-organization does not obey Newton's reductionism of (unphysical) point masses without an inside heat-energy or other internal degrees of freedom. The increase of the measurable (coordinate) speed at the geodesic take-off from the relativistically strong field is accompanied by the decrease of the shared interference energy that balances thermokinetic dynamics without pseudo-scientific mysteries. The local metric fields of distributed material densities are self-organized in a way to exhibit the Newton acceleration of inverse squares at the weak-field periphery and the pulsating acceleration/deceleration next to the strong-field central region. Poincaré first inferred the need in such adaptive tensions [10, 11] within the stable extended charge to compensate the formal repulsion by Coulomb's distant forces.

Dual classical physics (of localized matter and delocalized fields) was satisfactorily applied only to the 
macroscopic world and the megacosmos. Physicists tend to assign non-dual densities of the same matter mainly to the microscopic (quantum) world, where dense energies tend to repeal each other instead of Newton's gravity. Two different ontologies of one material world cannot coexist. The unique physical reality is either dual or non-dual, regardless of spatial scaling and mathematical formalisms in accessible theories. Similarly, inertial matter is either local or non-local, regardless of suitable approaches to its description. Today, researchers tend to consider spatial nonlocality of a closed system beyond quantum approaches and SR restrictions for retarded energy exchanges between independent systems [12, 13. New possibilities of holism [14] for macroscopic physics and the global instantaneous overlap of all material elements in quantum physics motivates our attempts to develop nonlocal thermokinetics for the inertial volume of rest-energy (which we call the inside heat capacity). There are no mesoscopic scales in modern physics for reasonable transitions from nondual microscopic states to the dual macroscopic model of Newton. Any material state should be considered through nondual, monistic terms of kinetic densities on the submicro-, micro-, macro-, and mega-scales at least in qualitative approaches if quantitative ones have not yet been found.

The monistic Cartesian reality of positive (yang) kinetic densities should not in principle rely on negative (yin) energies. In order to remove fictional (yin) notions from the relativistic self-organization of positive energies, it is necessary to rethink all known observations and modern experiments, including relativistic corrections to Newton's theory, in suitable terms of positive energies (competing for kinetic degrees of freedom). Such a radical renovation can be completed for the Cartesian matter-extension and its pushing self-tensions next to visible vertexes of continuously distributed inertia. We will examine Einstein's metric formalism in corresponding terms of mass-energy densities and will find below that bi-vertex degrees of freedom are competing with mono-vertex ones for the nonlocal kinetic energy of the multi-vertex unity. Nonlocal thermal space-matter with bi-vertex topology of interference energies can adaptively push inertial densities of metric matter associated in observations with heavy mechanical volumes. All continuous players, including thermokinetical waves or mechanical photons, carry only positive energies despite geodesic attractions and repulsions of probe bodies in the closed metric organization. The two-fraction competition of positive kinetic energies with bi-vertex and mono-vertex densities can quantitatively describe the thermomechanical Universe monistically through the kinetic distribution of nonlocal inertia without the need for auxiliary notion of negative (yin) gravitational potentials.

The purpose of this paper is to present the self-contained thermokinetic theory of nonlocal inertia with its instant self-coordination or correlation over a closed system. Based on the ideas of Aristotle [8], Descartes [6, 7], Clifford [15], Mie [16], Einstein and co-authors [17, 18], and the author [3, 19, 20], 
it is possible to rewrite the metric space-time in purely field terms of inertial densities, that is, without localized inertial particles. We start from the 1938 Einstein-Infeld statements and replace the supposed empty space (between formally localized charges) by the Cartesian non-empty space of material fields with distributed densities of active and passive mechanical charges (equal in static equilibrium). This can assist to in giving up the unnecessary concept of Newton's point-like particles and to modernize the unfinished Einstein's geometrization of material densities (or inertial fields) by singularity free solutions of metric. This theory enables adaptive states in external fields and wave signals from other systems. The hierarchy of subsystems and upper-systems can shed some light on the non-equilibrium dynamics of self-pulsating energy elements, warm matter self-assembling into ordered structures and living system organization. We will try to identify non-Newtonian chaos-order causes of cyclical pulsations in the inertial densities of material spaces. We will also propose verification/falsification procedures for self-organization of positive kinetic energies in multi-vertex inertial distributions in order to support the chaos-order self-governance and the ninlocal thermokinetic origin of local metric forces in the warm material space. In particular, the monistic mechanics of thermokinetic material space without fictional gravitational pulls of negative energies can make a testable quantitative prediction regarding the accelerated expansion of the dense nonlocal Metagalaxy and the periodic pulsations of other non-equilibrium self-organizations with dense space-matter regions.

\section{Bi-vertex interference in nonlocal energy distributions}

Now we begin with a brief look at the non-empty space interpretation [19, 20, 21] of the pseudoRiemannian space-time in applications to the multi-body mechanical problem. The latter remains practically unresolved in the available relativistic generalization [9] of Newtonian gravitation in empty (and curved) 3-space. In our approach, the non-empty space gains the interference, thermal energy under the mutual accelerations of mono-vertex elements. The latter loss, therefore, a part of Hamilton's kinetic energy to keep the system energy steady. The shared material space is a missed reservoir of kinetic energy deposits, which corresponds to the virial theorem losses. Interference inertial densities form this shared space which in turn pushes the mono-vertex carriers of inertia. In this self-coordinated organization of energy, the mechanical system of continuous mono-vertex and bi-vertex (interference, dark) densities keeps its full integral $\sum m_{n} c^{2}=m_{s y s} c^{2}=$ const regardless of the metric dynamics of all inertial elements. The visible interaction of mono-vertex elements within the nonlocal mechanical system with the continuous inertial density of its relativistic mass-energy was interpreted by Newton as the 
action-at-a-distance. The bi-vertex, interference energy content can be inferred, in principle, due to local tensions of the shared material space, its non-equilibrium thermal properties and measurable wave excitations. The mono-vertex fraction of the positive inertial energy or the heat-chaos capacity of the inside variable, $k m_{n} c^{2} \equiv \sqrt{1-\beta^{2}} m_{n} c^{2}$, can also be inferred from the Lorentz transformations of heat, $Q=Q_{o} \sqrt{1-\beta^{2}}$. Indeed, the ordered spatial transport of chaotic elements reduces the inside mass-energy density, $k \mu c^{2} \Rightarrow\left(1-0.5 \beta^{2}\right) \mu c^{2}$, or the inertial heat capacity which Umov estimated by the dimensionless variable $0.5 \leq k \leq 1$ in 1873 [22]. These Umov's losses of the inside rest-energy will be extracted to kinetic energy of the shared space, which below will participate as a material partner in our two-fractional virial theorem. The inside energy variable $k(\beta) m c^{2}$ of Umov, not a non-physical transport of the allegedly constant rest-energy $m c^{2}$, enables the adaptive chaos-order dynamics [3] in General Relativity and metric self-organization of inertia with adaptive thermokinetic energies.

The multi-vertex solution $g_{o o}^{-1 / 2}(x)=1+\sum \varphi_{i}(x) / \varphi_{G}$ for static metric fields was already found from the equivalence of equilibrium densities of active and passive mechanical charges [20, 21]. The constant $\varphi_{G} \equiv c^{2} / \sqrt{G}=1.04 \times 10^{27} \mathrm{eV}$ is the energy-information bandwidth for the mechanical charge, $\sqrt{G} m_{a} \varphi_{G}=m_{a} c^{2}$. All equilibrium spaces universally take the Euclidean 3 -section, $\gamma_{i j} \equiv g_{o i} g_{o j} g_{o o}^{-1}-g_{i j}=$ $\delta_{i j}, g^{i j}=-\delta^{i j}$, and $\sqrt{-g} \Rightarrow \sqrt{g_{o o} \gamma}=\sqrt{g_{o o}(x)}[19,20]$. For the sake of mathematical simplicity of the metric solutions for continuous inertial densities, we study the multi-vertex material space in the absence of rotations, $g^{o i}=g_{o i}=0$. The GR connections, $\Gamma_{\mu \nu}^{\nu} \equiv \partial_{\mu} \ln \sqrt{-g}$, of the pseudo-Riemannian spacetime can describe non-vanishing field intensities, $E_{\mu} \equiv-\varphi_{G} \Gamma_{\mu \nu}^{\nu}=-\varphi_{G} \Gamma_{\mu o}^{o}=\varphi_{G} \partial_{\mu} \ln \left[1 / \sqrt{g_{o o}}\right]=-\partial_{\mu} W$ through 4-gradients of one metric potential $\varphi_{G} \ln \sqrt{g_{o o}(\mathbf{x})} \equiv W(\mathbf{x})$ in the static multi-vertex space of inertial matter. The radial motion of inertial densities with the spherical symmetry are free from 3potentials or 'gravi-magnetic' fields. Such a simplified symmetrical motion can clarify (without the loss of generality) the relativistic chaos-order self-organization for static and self-pulsating material spaces of continuous energies. Active $\left(\sigma_{a}\right)$ and passive $\left(\sigma_{p}\right)$ charge densities of the equilibrium material space maintain the Einstein Principle of Equivalence and Euclidean 3D geometry:

$$
\sigma_{a}(x) \equiv \frac{\delta^{i j} E_{i}(x) E_{j}(x)}{4 \pi \varphi_{G}}=-\frac{\delta^{i j} \partial_{i} E_{j}(x)}{4 \pi} \equiv \sigma_{p}(x) .
$$

The strong field solution for the affine potential $W(x)$ of the multi-vertex material space [20],

$$
W(x) \equiv-\varphi_{G} \ln \frac{1}{\sqrt{g_{o o}(x)}}=-\varphi_{G} \ln \left(1+\frac{r_{1}}{\left|\mathbf{x}-\mathbf{a}_{1}(t)\right|}+\frac{r_{2}}{\left|\mathbf{x}-\mathbf{a}_{2}(t)\right|}+\ldots+\frac{r_{n}}{\left|\mathbf{x}-\mathbf{a}_{n}(t)\right|}\right),
$$

equalizes active and passive charge densities in (1). The GR Equivalence Principle becomes from (1)-(2) a mathematical theorem in the present self-organization of continuous material densities with Euclidean 
3D geometry. Hereinafter $t \equiv x^{o} / c$ is the universal time parameter for the multi-vertex metric world. The active charge density $\sigma_{a}(x)$ possesses the positive (inertial, kinetic) energy density $\mu_{i n}(x) c^{2}=\sigma_{a}(x) \varphi_{G}$. The elementary scale in the affine potential, $r_{k} \equiv E_{k} / \varphi_{G}^{2}$ is proportional to the positive (kinetic) restenergy $E_{k}^{G R}=q_{k} \varphi_{G}>0$ of the active (inertial) charge $q_{k}>0$. The elementary charge $q_{k}$ is continuously distributed almost radially everywhere, but mainly in the vicinity of the moving vertex $\mathbf{a}_{k}(t)$. Each elementary inertial charge $q_{k} \equiv E_{k}^{k i n} / \varphi_{G}$ keeps its value and, consequently, its elementary kinetic energy $E_{k}^{k i n}=m_{k} c^{2} \sqrt{g_{o o}^{\neq k}\left(\mathbf{a}_{k}[t]\right)} / \sqrt{1-\beta_{i}^{2}(x)}>0$ on the geodesic paths (in the absence of inelastic or wave exchanges) in constant external fields. Notice that GR metric function $\sqrt{g_{o o}^{\neq k}}$ does not create contributions from negative (non-existing, yin) energies, but only changes positive (yang, existing) kinetic energies of inertial bodies. In yin-yang physics of Chinese parallel worlds, Einstein did geometrize the SR kinetic energy of corporeal matter but not negative potential energies of Newton from the incorporeal world. In this way, the yin-yang dialectical heritage can support Kuhn's conflict of paradigms in modern mechanics [1] by clarifying where Einstein's metric theory of always positive SR energies conceptually disagrees with the error model of negative potential energies of Newton.

The mono-vertex active and passive densities in $(1)$ can take the static radial solution $[19] \sigma_{a}(r) \varphi_{G}=$ $\sigma_{p}(r) \varphi_{G}=\varphi_{G}^{2} r_{m}^{2} / 4 \pi r^{2}\left(r+r_{m}\right)^{2}=\mu c^{2}$ at the Euclidean section of the four-interval $d s^{2}=g_{o o} d x_{o}^{2}-$ $\delta_{i j} d x^{i} d x^{j}$, and $g_{o o}=r^{2} /\left(r+r_{m}\right)^{2}$. The strong-field logarithmic potential (2) defines the energy density $\sigma_{a}(x) \varphi_{G}$ in the multi-vertex inertial space. One can integrate the left hand side of (1) analytically (by making use from the mathematical equality with the right hand side) in order to find the kinetic (active, yang) energy of the multi-vertex system:

$$
\begin{aligned}
& E_{\text {sys }}^{k i n}=\int \frac{c^{4} d^{3} x}{4 \pi G}\left(-\frac{\frac{\left(\mathbf{x}-\mathbf{a}_{1}\right) r_{1}}{\left|\mathbf{x}-\mathbf{a}_{1}\right|^{3}}+\frac{\left(\mathbf{x}-\mathbf{a}_{2}\right) r_{2}}{\left|\mathbf{x}-\mathbf{a}_{2}\right|^{3}}+\ldots+\frac{\left(\mathbf{x}-\mathbf{a}_{n}\right) r_{n}}{\left|\mathbf{x}-\mathbf{a}_{n}\right|^{3}}}{1+\frac{r_{1}}{\left|\mathbf{x}-\mathbf{a}_{1}\right|}+\frac{r_{2}}{\left|\mathbf{x}-\mathbf{a}_{2}\right|}+\ldots+\frac{r_{n}}{\left|\mathbf{x}-\mathbf{a}_{n}\right|}}\right)^{2}=\int \frac{\varphi_{G}^{2} d^{3} x}{4 \pi} \frac{\frac{r_{1}^{2}}{\left|\mathbf{x}-\mathbf{a}_{1}\right|^{4}}+\frac{r_{2}^{2}}{\left|\mathbf{x}-\mathbf{a}_{2}\right|^{4}}+\ldots+\frac{r_{n}^{2}}{\left|\mathbf{x}-\mathbf{a}_{n}\right|^{4}}}{\left(1+\frac{r_{1}}{\left|\mathbf{x}-\mathbf{a}_{1}\right|}+\frac{r_{2}}{\left|\mathbf{x}-\mathbf{a}_{2}\right|}+\ldots+\frac{r_{n}}{\left|\mathbf{x}-\mathbf{a}_{n}\right|}\right)^{2}} \\
& +\sum_{i=1 k \neq i}^{n} \sum^{n-1} \int \frac{\varphi_{G}^{2} d^{3} x}{4 \pi} \frac{\frac{\left(\mathbf{x}-\mathbf{a}_{1}\right) r_{i}}{\left|\mathbf{x}-\mathbf{a}_{1}\right|^{3}} \times \frac{\left(\mathbf{x}-\mathbf{a}_{2}\right) r_{k}}{\left|\mathbf{x}-\mathbf{a}_{2}\right|^{3}}}{\left(1+\frac{r_{1}}{\left|\mathbf{x}-\mathbf{a}_{1}\right|}+\frac{r_{2}}{\left|\mathbf{x}-\mathbf{a}_{2}\right|}+\ldots+\frac{r_{n}}{\left|\mathbf{x}-\mathbf{a}_{n}\right|}\right)^{2}}=E_{m o}^{k i n}(t)+E_{b i}^{k i n}(t)=\varphi_{G}^{2} \sum r_{i}=\varphi_{G} \sum q_{i} .
\end{aligned}
$$

For the constant sum $\sum r_{i}=$ const, this integral claims the conservation law for the system inertial charge $q_{\text {sys }}=\sum q_{i}=\varphi_{G} \sum r_{i}=$ const and the conservation law for the system rest-energy $E_{\text {sys }}^{\text {kin }} \equiv$ $E_{m o}^{k i n}+E_{b i}^{k i n}=\varphi_{G} q_{s y s}=$ const $>0$ despite arbitrary positions $\mathbf{a}_{i}(t)$ of its poles. Elastic systems also have the conservation of their elementary energies, $\varphi_{G} q_{i}=$ const. Inelastic exchanges of wave radiation (mechanical photons) can change elementary energies, $\varphi_{G} q_{i} \neq$ const, under the system conservation $\sum \varphi_{G} q_{i}=$ const. Due to the system charge-energy conservation under the inside exchanges, we can call a closed system (with even non-equilibrium densities) an elementary one and consider it again as one mono- 
vertex element with the inside heat-energy capacity in a larger system of the world matter hierarchy. This is the ground for a metric organization of quasi-equilibrium sub-systems with short sub-times into heavy systems with larger times for multi-level equilibrium formations due to parallel dissipative exchanges in the shared space-matter.

The passive charge density $\sigma_{p}(x)>0$ in the system metric potential (2) provides the negative local density $\sigma_{p}(x) W(x)<0$ to the passive (yin) energy integral $E_{s y s}^{p o t}<0$. This relativistic potential (yin) self-energy generalizes the potential self-energy of Newton like the relativistic kinetic (yang) self-energy $E_{\text {sys }}^{k i n}>0$ generalizes the classical kinetic energy $m V^{2} / 2$. Newtonian gravitational self-energy diverges in the field center of the point source and this negative self-energy is not handled in textbooks for massless fields around point matter. The negative (gravitational, yin) self-energy cannot be in principle measured in the physical reality of positive, kinetic energies where only active (inertial, yang) energies can be detected under mechanical collisions or heat exchanges. However, we calculate the spatial volume integral $E_{s y s}^{p o t}=\int \sigma_{p}(x) W(x) d^{3} x<0$ in order to derive theoretically the immeasurable (negative, yin) self-energy of the multi-element system:

$$
E_{\text {sys }}^{\text {pot }}=\int \sigma_{p}(x) W(x) d^{3} x=\int \frac{W(x) \nabla^{2} W(x)}{4 \pi} d^{3} x=-\int \frac{[\nabla W(x)]^{2}}{4 \pi} d^{3} x=-\varphi_{G} \sum q_{i} \equiv-E_{\text {sys }}^{k i n} .
$$

It turns out that the positive (kinetic) and negative (potential) self-energies (3) and (4), respectively, are compensated with the exact algebra symmetry, $E_{s y s}^{k i n}+E_{s y s}^{p o t} \equiv 0$, for any number of vertexes inside a considered system. The universal compensation (4) means that the visible (corporeal) world of inertial (kinetic, measurable) energies is formally accompanied by the invisible (incorporeal) world of negative (potential, immeasurable) energies. The similar yin-yang conjugation takes place for imaginary electric charges [21].

The active and passive integrals in (4) are identical for closed systems of metric energies. This yinyang mathematical symmetry between positive (kinetic) and negative (potential) energies does not mean physical interactions or energy exchanges between elements of corporeal and incorporeal worlds as mirror images in optics do not interact with their real objects. One should assume mutual interactions of only positive kinetic energies in (3) in order to understand the physical reality, which is the corporeal world in Chinese philosophy. Below we require the conservation of positive kinetic energies (3) for a closed system. Newton did consider bodies without conserving of their summary kinetic energy and used, in fact, only the auxiliary symmetry of yin-yang energies rather than the unknown relativistic conservation of purely kinetic energies. But how can one discard in General Relativity the non-physical (yin, negative) potentials (4) from further considerations of these 'purely kinetic energies' without contradicting to the 
phenomenon of gravitational pulls in the observable (yang) practice?

\section{Virial losses of mono-vertexes and supplementary warming of bi-vertex densities in the shared material space}

It seems a bit surprising for physics with Newtonian negative potentials that the metric energy functional (3) does not depend on instant spatial positions $\mathbf{a}_{i}(t)$ of geometrical centers of elementary energy distributions, involved in mutual metric interactions. Such a system energy conservation of overlapping elements can take place due to positive contributions to the kinetic (yang) energy $E_{\text {sys }}^{k i n}$ from non-Newtonian bi-vertex or interference formations of continuous positive densities in (3). Indeed, the constant system energy (4) and its inertial elementary charge $q_{s y s}^{a}=E_{s y s}^{k i n} / \varphi_{G}=$ const can be represented by the sum of two time-varying topological charges, $q_{m o}(t)+q_{b i}(t)=q_{s y s}^{a}=E_{s y s}^{k i n} / \varphi_{G}=$ const. If vertexes $\mathbf{a}_{1}, \mathbf{a}_{2}, \ldots \mathbf{a}_{n}$ of continuous monopoles are separated by infinite distances then there are no bi-vertex charges in such an initial system. The system thermal (kinetic) capacity belongs only to the inside relativistic energy, while the outside thermal energy is equal to zero. One can say for this limit that the shared material space and its kinetic energy (or Maxwell temperature) are vanishing. Such a cold static system has maximum of its outside order and maximum of its inside (hidden) heat-chaos. The shared material space and its self-warming is related to partial extractions of Umov's inside energy from interacting monopoles. The shared space is filled continuously by bi-vertex densities of inertial (yang) energy or elementary topological bipoles.

The energy integral (3) for densities $\left(\sum a_{i}\right)^{2} \Rightarrow \sum a_{i}^{2}$ of monopoles with vanishing interferences, $\sum_{1}^{n}\left(a_{i} \sum_{k \neq i}^{n-1} a_{k}\right) \Rightarrow 0$, can be calculated directly. Rapprochement of two distant vertexes split the volume integration in (3) over $n$ mono-vertex densities, $\propto r_{i}^{2}\left|\mathbf{x}-\mathbf{a}_{i}\right|^{-4}$, and (n-1) interference or bi-vertex densities, $\propto r_{i} \sum_{k \neq i}^{n-1} r_{k}\left|\mathbf{x}-\mathbf{a}_{i}\right|^{-2}\left|\mathbf{x}-\mathbf{a}_{k}\right|^{-2}$. Now, the constant kinetic energy (3) of the multipolar system contains the time-varying energies of inertial monopoles and the time-varying energies of inertial bipoles. The latter form the shared, interference space with material densities and their local tensions. Both mono-vertex and bi-vertex topological charges have positive (kinetic) densities. Together they contribute together to the summary inertial charge $E_{s y s}^{k i n} / \varphi_{G}=$ const of a closed (elementary) system. Time-varying bi-vertex deposits are known from the interference phenomena in quantum mechanics and in optics, but not in Newtonian mechanics of point particles without mutual overlaps. Unlike the concentrated mono-vertex energy of visible in practice monopoles, bi-vertex energies were not measured directly. The bi-vertex fraction of mass-energy can be called the dark energy. 
The need to add bi-vertex contributions of the massive interference fields into the GR rest mass of the two-body system was first claimed by Brullouin in his new look at the relativistic theory [23]. This correct, but under-appreciated view was considered at that time a criticism of General Relativity rather than a reasonable attempt for theoretical improvements. The positive bipolar energy is, in fact, the extraction of the inside heat-energy of inertial charges into their joint interface or the shared material space. By neglecting the 1970 comments of Brillouin, modern relativistic physics neglected the shared inertial space of interference energies. The kinetic energy absorptions by the inside rest-energy variable, but not the unknown 'dark energy' mechanisms, will be used by us below for the geodesic cooling and self-acceleration of the expanding space-matter in the Cartesian Metagalaxy.

Again, if vertexes $\mathbf{a}_{i}$ of radial monopoles of inertial matter are separated into infinite distances, then negative gravitation shifts of the monopole energies vanish. Bi-vertex interference deposits to inertia of this system vanish as well. Analytical integration in (3) yields directly $\varphi_{G} \sum q_{i}$ for infinitely remote monopoles. They can be observed in practice as localizations of separated carriers of inertia without the measurable temperature. Local observations of remote elements cannot clarify all their bounds within a single material system (or within the non-local oneness of the elementary system). If vertexes of monopoles are separated by finite distances, then the same system energy (3) contains diminished contributions from interacting monopoles together with increased contributions from arising bipole formations or bi-vertex topological charges.

Notice that remote observations of elementary particles (dense material spaces of continuous inertial energy) as independent objects misrepresent their mechanical system as a solo number of these objects without new material integrations. In fact, the single whole is qualitatively greater than the initial sum of individual monopoles. There are bi-vertex formations with elastic stresses and inelastic wave excitations which are qualitatively new topological entities with their quantitative characteristics. Bi-vertex topological charges have finite inertial inputs into elementary inertial charges. In practice, such nonlocal topological charges cannot be extracted from the system for a remote investigation and measurements. Because of bipoles with their dark energy, the inertial (kinetic) energy of neighboring monopoles is always less than their inertial rest-energy outside of the system. The latter split the elementary rest-energy into mono-vertex and bi-vertex parts. One can say that monopoles moving in relations to each other are losing their inside heat energies by extracting it into the shared material space. Time-varying bipoles are gaining positive (dark) energies on bi-vertex degrees of freedom that form the nonlocal space-matter with local tensor tensions. These tensions generate the local 4-force of geodesic pushes that reveals the kinetic nature of the 1st fundamental force for nonlocal interactions between dense regions of metric 
space-matter.

By operating with bi-vertex energies, which are nonlocal topological formations, one can reiterate many quantitative conclusions of Bohm 24] regarding the wholeness of the undivided Universe. Below we try to develop a quantitative approach to the multi-vertex densities in the nonlocal oneness. At first, we separate the inertial energy of monopoles analytically from the indivisible sum of mono-vertex and bi-vertex inertial energies within their joint inertial matter. In order to bypass computer simulations, we consider in (3) only relatively large distances $R_{i k}(t) \equiv\left|\mathbf{a}_{k}(t)-\mathbf{a}_{i}(t)\right|>>r_{i}+r_{k}$ between centers of almost radial monopoles. The volume integration in (3) of almost radial densities $r_{i}^{2}\left|\mathbf{r}-\mathbf{a}_{i}(t)\right|^{-4}$ (without $r_{i} r_{k}\left|\mathbf{r}-\mathbf{a}_{i}(t)\right|^{-2} \times\left|\mathbf{r}-\mathbf{a}_{k}(t)\right|^{-2}$ bi-vertex contributions) can provide the analytical approximation for the positive time-varying energy of monopoles,

$$
\begin{aligned}
& E_{m o}(t) \approx \int \frac{d^{3} x}{4 \pi} \frac{\varphi_{o}^{2} r_{1}^{2}}{|\mathbf{x}|^{4}\left(1+\frac{r_{1}}{|\mathbf{x}|}+\frac{r_{2}}{\left|\mathbf{a}_{1}-\mathbf{a}_{2}\right|}+\ldots+\frac{r_{n}}{\left|\mathbf{a}_{1}-\mathbf{a}_{n}\right|}\right)^{2}}+\int \frac{d^{3} x}{4 \pi} \frac{\varphi_{o}^{2} r_{2}^{2}}{|\mathbf{x}|^{4}\left(1+\frac{r_{1}}{\left|\mathbf{a}_{2}-\mathbf{a}_{1}\right|}+\frac{r_{2}}{|\mathbf{x}|}+\ldots+\frac{r_{n}}{\left|\mathbf{a}_{2}-\mathbf{a}_{n}\right|}\right)^{2}} \\
& +\ldots+\int \frac{d^{3} x}{4 \pi} \frac{\varphi_{o}^{2} r_{n}^{2}}{|\mathbf{x}|^{4}\left(1+\frac{r_{1}}{\left|\mathbf{a}_{n}-\mathbf{a}_{1}\right|}+\frac{r_{2}}{\left|\mathbf{a}_{n}-\mathbf{a}_{2}\right|}+\ldots+\frac{r_{n}}{|\mathbf{x}|}\right)^{2}}=\sum_{i=1}^{n} q_{i} \varphi_{G} \sqrt{g_{o o}^{\neq i}(t)}=\sum_{i=1}^{n} q_{i}^{2} \varphi_{o}^{2} \sqrt{1-\beta_{i}^{2}(t)} / m_{i} c^{2} \\
& \approx \sum_{i=1}^{n} q_{i}\left(\varphi_{G}-\sum_{k \neq i}^{n} \frac{q_{k}}{R_{i k}(t)}\right) \approx \sum_{i=1}^{n} m_{i}\left(c^{2}-\frac{v_{i}^{2}(t)}{2}\right)
\end{aligned}
$$

where an element $q_{i}$ of the system charge 'feels' the external for it (specific) metric $g_{\text {oo }}^{\neq i}$, rather than the system metric component $g_{o o}$ in (2). The item $q_{2} \varphi_{G} \sqrt{g_{o o}^{\neq 2}\left(\mathbf{a}_{2}\right)} \equiv q_{2} \varphi_{G} /\left(1+\frac{r_{1}}{\left|\mathbf{a}_{2}-\mathbf{a}_{1}\right|}+\frac{r_{3}}{\left|\mathbf{a}_{2}-\mathbf{a}_{3}\right|}+\ldots+\frac{r_{n}}{\left|\mathbf{a}_{2}-\mathbf{a}_{n}\right|}\right)$, for example, contains negative shifts of the positive inside energy $q_{2} \varphi_{G}$, associated with paired interactions of $q_{2}$ with all other elements $q_{k \neq 2}$. These small shifts of active (yang) kinetic energies by negative (yin) potentials might correspond to the symmetrical inclusions of two opposite black and white circles in the Yin-Yang Symbol. This bi-color reading of four related elements also describes the connected 'being' and 'not-being' with the symmetrical 'generation' and 'destruction' in Aristotle's Metaphysics [8]. Thus, the Yin-Yang Symbol of Chinese thinkers should be integrated into the relativistic physics of variable Umov's energies. In accordance with the Yin-Yang Symbol, GR calculations should be oriented only on the inertial (corporeal, yang) segment of thermokinetic energies with small metric changes of their positive values. Ultimately, GR with the Umov's inside variable becomes a theory of purely kinetic energies in terms of metric inertia rather than metric gravity.

After a long process of elastic self-pulsations with relatively slow dissipative damping, an isolated massenergy $q_{s y s} \varphi_{G} \equiv r_{s y s} \varphi_{G}^{2}=$ const can form the equilibrium self-organization as one radial monopole with the static densities $\varphi_{G}^{2} r_{\text {sys }}^{2} / 4 \pi r^{2}\left(r+r_{\text {sys }}\right)^{2}$ [19]. Each inertial charge tends to adaptive self-organization of its material densities in own metric fields and in external fields. The elementary mass-energy integral 
of the extended charge moves in external fields as one probe body in the absence of radiation losses; this elastic motion obeys the GR geodesic law. A 'probe body' motion in specific external fields means that GR should operate with a specific gravitational time dilation for each elementary object, a specific fourinterval $\sqrt{d \tau_{i}^{2}-d l^{2}}$, and specific $4 \mathrm{D}$ pseudo-Riemannian geometry in the absence of one common time sub-interval, $d \tau_{i} \neq d \tau_{k}$. However, the spatial sub-interval $d l$ can have the universal (Euclidean) geometry for all sub-systems. Only universal 3D sub-geometry provides each elementary space and local observers the same universal ruler for 3D scales and length measurements. Any inertial body is moving in its unique and curved 4D space-time with a specific external field. All these 4D manifolds have the common (world) and flat (universal) 3D space under the join world parameter $x^{o} / c=t[25$ ]. For such a specific organization of the GR geodesic dynamics, with the elastic path conservation $m_{i} c^{2} \sqrt{g_{o o}^{\neq i}(t)} / \sqrt{1-\beta_{i}^{2}(t)}=$ const of the inertial charge in constant external fields, one should redefine the potential energy of paired interactions compared to the conventional potential energies in the Newton theory. Newton shared one potential energy $-G m_{1} m_{2} / R$ between both bodies in the classical conservation law, $\left(m_{1} V_{1}^{2}+m_{2} V_{2}^{2}\right) / 2-$ $G m_{1} m_{2} / R=$ const. Our concept of the elastic (geodesic) motion in the external potentials and the interference (bi-vertex, dark) energy extraction $q_{1} q_{2} / R$ into the shared inertial space requires another reading of the two-body system, $\left[m_{1}\left(c^{2}-G m_{2} / R\right)+m_{1} V_{1}^{2} / 2\right]_{m o}+\left[m_{2}\left(c^{2}-G m_{1} / R\right)+m_{2} V_{2}^{2} / 2\right]_{m o}+$ $\left(G m_{1} m_{2} / R\right)_{b i}=\left(m_{1}+m_{2}\right) c^{2}=$ const. This physics of visible monopoles and dark bipoles in their shared material space with its degree of energy freedom explains in (3) the field energy density $\mathbf{D}^{2} / 4 \pi$ (instead of $\mathbf{D}^{2} / 8 \pi$ ) for both thermomechanics and electrodynamics of extended complex charges, where $\varphi_{G} \operatorname{div} \mathbf{D}=-4 \pi\left(\epsilon_{m} \pm i\left|\epsilon_{e}\right|\right)[26,21]$.

The value of inertial elementary charges is proportional to their yang (positive) energy, $q_{i}(x)=$ $E_{i}(x) / \varphi_{G}=m_{i} c^{2} \sqrt{g_{o o}^{\neq i}\left(\mathbf{a}_{i}[t]\right)} / \sqrt{1-\beta_{i}^{2}(t)}$. This charge is not constant for the case of motion with inelastic collisions or radiation exchanges. One can consider the time-averaged motion of interacting monopoles in the closed system, when $\overline{q_{i}(t)} \varphi_{G}=m_{i} c^{2} \sqrt{g_{o o}^{\neq i}(t)} / \sqrt{1-\beta_{i}^{2}(t)}=m_{i} c^{2} \lambda_{i}$. Thus, by averaging over time $t$ the summary energy of monopoles in (5), where $\overline{g_{o o}^{\neq i}(t)}=\lambda_{i}^{2}\left[\overline{1-\beta_{i}^{2}(t)}\right]$, we can derive the relativistic analog of the classical virial theorem for the finite periodical motion,

$$
\overline{E_{m o}(t)}=\varphi_{G} \sum_{i=1}^{n} \overline{q_{i} \sqrt{g_{o o}^{\neq i}\left(\mathbf{a}_{i}[t]\right)}}=\sum_{i=1}^{n} m_{i} c^{2} \lambda_{i}^{2} \overline{\sqrt{1-\beta_{i}^{2}(t)}} \Rightarrow \sum_{i=1}^{n} m_{i}\left(c^{2}-\frac{\overline{v_{i}^{2}(t)}}{2}\right) .
$$

The positive parameter $\lambda_{i} \leq 1$ depends on initial static positions of $q_{i}$ if its kinetic energy in infinity is defined as $m_{i} c^{2}$. The weak field start corresponds $\lambda_{i} \Rightarrow 1$, if all centers $\mathbf{a}_{i}$ of interacting massenergies $E_{o i}=m_{i} c^{2}$ were separated before the free motion by large distances (much above $\sum E_{o i} / \varphi_{G}^{2}$ ). The relativistic virial theorem $(6)$ is better known for its non-relativistic limit of slow particles. It 
demonstrates a very unique phenomenon: the faster the cyclic motion of visible monopoles, the less is their averaged energy in Newtonian mechanics. In reality, there are kinematic losses of inside massesenergies $\mu m c^{2} \leq m c^{2}$ of Umov even for the nonrelativistic motion. These losses correspond to the decreased capacity of inside heat-chaos $Q=Q_{o} \sqrt{1-\beta^{2}}$ (like the decreased energy $\hbar \omega=\hbar \omega_{o} \sqrt{1-\beta^{2}}$ of the quantum particle) of a moving one-vertex element of inertia under the Lorentz rules for proper time dilation [3].

The energy sum $\sum E_{i} \sqrt{g_{o o}^{\neq i}(t)}>0$ of monopole formations is, in fact, the inside heat-chaos of Umov which can be extracted to the shared space due to mutual interactions of inside heat-energies and the ordering of chaos under its spatial motion. The metric tensor component $g_{\text {oo }}$ defines in (3), (5), and (6) exclusively kinetic (positive) energies which obey metric interactions under their relativistic self-organization. The physical reality is the world of positive, inertial energies with the kinematic restrictions of Special Relativity for separated elementary systems. The metric theory of one nonlocal self-organizations drives instant kinetic reasons for the adaptive motion of the system densities to their equilibrium. The bi-vertex fraction of energy densities in (3) or the positive (yang) interference energy describes the shared inertial space as the self-organization of kinetic energies in a closed nonlocal system. The sum $\sum E_{i}\left[1-\sqrt{g_{o o}^{\neq i}(t)}\right]>0$ of bi-vertex energies is the time-varying kinetic energy of the warmed material space in relativistic thermomechanics. This positive energy, extracted from the inside energy of Umov, can be associated with thermodynamical functions and the measurable temperature of Maxwell.

The virial theorem in Newtonian physics looks incomplete without time-varying topological bipoles or dark mass-energy on the kinetic degrees of freedom of shared material space. Kinetic or thermal energy cannot escape the closed mechanical system and should be accumulated somewhere in the space of this system. In the physics of nonempty (material) space, the competition of inside and shared degrees of freedom drives self-accelerations / self-decelerations of mechanical bodies in metric fields [3]. Newtonian constant mass has no inside energy variables and this misleading notion should be replaced by the inside heat content in the dynamical problems of adaptive self-organization of kinetic chaos and order. General Relativity of extended inertial charges with the inside energy variable should count the shared space of nonlocal inertia as a dynamical partner (a kind of elastic or timeless neutrinos next to inelastic mechanical photons) to preserve the chaos-order balance and the conservation of kinetic energy (3) in cosmic collisions of inertial elements.

Negative potential shifts of positive (yang) energies of monopoles are balanced in (3) by complementary energies of nonlocal bipoles or the interference (dark) deposits $a_{i} \sum_{k \neq i}^{n-1} a_{k}$ to the thermokinetic energy 
$E_{b i}(t)$ of the shared material space,

$$
\begin{array}{r}
E_{b i}(t)=\sum_{i=1}^{n} \sum_{k \neq i}^{n-1} \int_{0}^{2 \pi} \frac{c^{4} d \varphi}{4 \pi G} \int_{0}^{\infty} r^{2} d r \int_{0}^{\pi} \frac{r_{i} r_{k}\left(r^{2}-R_{i k} r \cos \theta\right) \sin \theta d \theta}{r^{3}\left(R_{i k}^{2}+r^{2}-2 R_{i k} r \cos \theta\right)^{3 / 2}}=\sum_{i=1}^{n} q_{i} \varphi_{G}\left(1-\sqrt{g_{o o}^{\neq i}(t)}\right) \\
=\sum_{i=1}^{n} q_{i} \varphi_{G}\left(1-\frac{q_{i} \varphi_{G}}{m_{i} c^{2}} \sqrt{1-\beta_{i}^{2}(t)}\right) \approx \sum_{i=1}^{n} q_{i} \sum_{k \neq i}^{n-1} \frac{q_{k}}{R_{i k}(t)} \approx \sum_{i=1}^{n} \frac{m_{i} v_{i}^{2}(t)}{2} .
\end{array}
$$

It is worth recalling that only the non-empty space paradigm may accept the positive (active, yang) bi-vertex or dark energy (7) and the relevant space warming/cooling in General Relativity. The 1916 metric gravitation for the point matter is free from local material overlaps and operates with cold empty space without kinetic energy densities and a space temperature. One may say from (3), (5) and (7) that paired interactions of monopoles generate their shared space with bi-vertex topology of elementary energies borrowed from the inside energy of monopole topological spaces. Weak field attractions of visible monopoles come not from a coordinate decrease of negative Newtonian potentials, but from the thermodynamical tendency of each elementary inertial charge $q_{i}$ toward dynamical equipartition, $E_{i} \sqrt{1-\beta_{e q}^{2}}=E_{i} \beta_{e q}^{2} / \sqrt{1-\beta_{e q}^{2}}$, of its one-vertex and two-vertex energies on the available degrees of freedom. This kinematic principle of motion [3] can clarify why monopoles are attracting ('gravitating') in weak fields and repealing ('anti-gravitating') in strong ones despite that the summary kinetic energy (3) is independent from mutual replacements.

The system self-organization with mutual transformations of mono-vertex and bi-vertex kinetic energies is responsible for dynamical changes of distributed inertia (and internal radiation exchanges, in the most general case). Visible elastic accelerations obey the self-governance of positive thermokinetical players without the need of negative potentials from the incorporeal (yin) partners. Negative gravitational energies can only exit in mathematical models and in the fictional world of incorporeal bodies. The current attempt of Newtonian proponents to quantize the gravitational field with negative (non-existent) energy can be considered as a kind of pseudo-science. The correct way is to quantize the positive summary energy of mono-vertex and bi-vertex parts.

The quantum transformations of inside heat-energies (3) of monopoles together with bi-vertex thermal energies (5) of the shared space can be considered as a slow dissipative process toward equilibrium states of kinetic systems. The stationary equipartition of averaged mono- and bi-vertex energies, $\left.\sum_{i=1}^{n} m_{i} c^{2} \lambda_{i}^{2} \overline{\sqrt{1-\beta_{i}^{2}(t)}}=\sum_{i=1}^{n} m_{i} c^{2} \lambda_{i}^{2} \overline{11-\sqrt{1-\beta_{i}^{2}(t)}}\right]$ can occur for $\overline{\beta_{i}^{2}(t)}=3 / 4$ or $\overline{\left|v_{i}\right|} \approx 0.866 c$ and $\overline{\left|d x^{i}\right| / d t}=\overline{\left|v_{i}\right|} \sqrt{1-\bar{\beta}_{i}^{2}} \approx 0.433 c$. 


\section{Accelerated expansion of the nonlocal Metagalaxy with geodesic self-cooling}

In this section we find that the dynamical self-cooling of the shared material space with the enhanced bi-vertex (or dark in the modern terminology) fraction of kinetic energy accompanies the accelerated expansion of adaptive self-organizations in the strong field limit of non-equilibrium inertial states. Cartesian non-empty space with adaptive inertia and cyclical pulsations of continuous material densities reinforces new challenges for the nonlocal world organization and the laboratory thermokinetics of Umov's energy flows. Today, the Yin-Yang Dialectics, the Aristotle's continuum plenum for space, and Descartes's matter-extension are still considered by many relativists as useless philosophies that are not confirmed directly by modern observations and precise laboratory measurements. However, the point mass has already been falsified by many measurements with quantum devices. To be reinforced in modern physics of inertia, the Euclidean material space of equilibrium extended masses must not only support all 'gravitational' phenomena and subtle relativistic corrections [25] to the Newton theory, but also explain something beyond the possibilities of curved empty space with the mathematical black hole. Below we apply the Euclidean material space with bi-vertex (dark) energies to the self-cooling Metagalaxy, accelerated expansion of which has not been explained satisfactorily by the theories with empty and curved 3-spaces.

The observed radius of high density matter in the center of our galaxy is a bit larger than its Schawrzschild gravitational scale $2 r_{m} \equiv 2 R_{o}$. And visible radii of neutron stars are also a bit above $2 G m_{i} / c^{2}$ as is known from many observations. The static balance for equilibrium monovertex densities can explain why extremely dense matter can stay steady despite the textbook singularities for collapsing matter. Dynamical pulsations of inertial heat-energy on different degrees of freedom can be proposed for closed cosmic systems if one considers the Einstein-type thought experiment with symmetrical radial motion instead of circular one in the original formulation [27. After this 1939 experiment, Einstein finally denied for reality the metric singularity, proposed by Schwarzchild [28] and also by Droste [29], because bodies cannot move arbitrarily ("at least not faster than light" [27]).

Now let us consider equal inertial bodies in symmetrical falls toward the center of massive radial field. In non-empty space physics with the geodesic principle of equipartition for inside and outside kinetic energies, the decelerated fall next to the center will follow the initial Newtonian acceleration in weak fields [3]. Material space continuum in the GR metric formalism can keep Euclidean 3D section of curved 4D space-time due to six inherent symmetries [19] of the real world geometry. These symmetries are important, in particular, for the steady spatial transport of probe energies with their conservation. The GR geodesic equations of motion in curved pseudo-Riemann space-time with $0 \leq g_{o o} \leq 1$ and the flat $3 \mathrm{D}$ 
section, $g_{o i} g_{o j} g_{o o}^{-1}-g_{i j}=\delta_{i j}$, have been already derived [25] for strong static fields,

$$
\left\{\begin{array}{l}
\frac{d t}{d p}=\frac{1}{g_{o \circ}}, \frac{d p}{d s}=\frac{g_{o o} d t}{d s}=\frac{E_{m}}{m}=\text { const, } \quad r^{2} \frac{d \varphi}{d p}=J_{\varphi}=\text { const, } r^{2} \frac{d \varphi}{d s}=J_{\varphi} \frac{E_{m}}{m} \equiv L=\text { const } \\
\left(\frac{d r}{d p}\right)^{2}+\left(\frac{J_{\varphi}}{r}\right)^{2}-\frac{1}{g_{o o}}=-\frac{m^{2}}{E_{m}^{2}}, \quad\left(\frac{d r}{d s}\right)^{2}+\left(r \frac{d \varphi}{d s}\right)^{2}-\frac{E_{m}^{2}}{m^{2} g_{o \circ}}=-1 .
\end{array}\right.
$$

Here the inertial energy $E_{m}=$ const of the probe body and its angular momentum $J_{\varphi}=$ const are the first integrals of the motion under the changing parameter $p$.

Now we can apply (8) to the Einstein-type thought experiment for the symmetrical radial fall of two equal counter-partners from infinity on the massive body in the center of this 3-body system. We consider the coordinate motion of the center of the first (extended) body in the material fields of the second and third extended bodies, with $E_{1} / m_{1}=c^{2} \sqrt{g_{o o}^{\neq 1}} / \sqrt{1-v_{1}^{2} c^{-2}}=$ const $\Rightarrow 1$ and $d \varphi_{1} / d s_{1}=0, d s_{1}=$ $\sqrt{g_{o o}^{\neq 1}} c d t \sqrt{1-v_{1}^{2} c^{-2}}, v_{1}^{2}=\left(d r / \sqrt{g_{o o}^{\neq 1}} d t\right)^{2}, 1 / \sqrt{g_{o o}^{\neq 1}}=1+\left(G E_{2} / c^{4} 2 r\right)+\left(G E_{3} / c^{4} r\right) \equiv 1+\left(r_{o} / r\right)$. The last equation in (8) results in the radial speed $d r / d t= \pm c \sqrt{g_{o o}^{\neq 1}\left(1-g_{o o}^{\neq 1}\right)}$ for the free radial fall - takeoff with respect to the world time $t$ of a distant observer [3]. The corresponding radial acceleration-deceleration,

$$
\frac{d^{2} \mathbf{r}(t)}{c^{2} d t^{2}}=\frac{\mathbf{r}}{r}\left(\frac{1}{2}-g_{o o}^{\neq 1}\right) \frac{d g_{o o}^{\neq 1}}{d r}=\frac{\mathbf{r}\left(r_{o}^{2}+2 r_{o} r-r^{2}\right) r_{o}}{\left(r+r_{o}\right)^{5}}
$$

in the metric field of one consolidated center with $r_{o}=G\left(2 M_{3}+m_{2}\right) / 2 c^{2}$ describes the Newton gravitational pulls $-r_{o} c^{2} \mathbf{r} / r^{3}$ for $r_{o} \ll r$ and the strong-field inertial pushes $+\mathbf{r} c^{2} / r_{o}^{2}$ for $r \ll r_{o}$ toward their equilibrium. The free fall acceleration in (8) takes its extreme value $-9.2 \times 10^{-3} c^{2} / r_{o}$ at $4.48 r_{o}$, while the fall deceleration takes its maximum $+0.12 c^{2} / r_{o}$ at $0.35 r_{o}$. The maximum coordinate (observable) speed $d r / d t=c / 2$ is predicted at the equilibrium radial proximity $r=r_{o}(1+\sqrt{2})$ to the center. Passing this kinematic equilibrium for inside and outside kinetic energies, when $d r / d t=c / 2$ and $d^{2} r / d t^{2}=0$, elementary inertial charges move with the deceleration to the center and stop here at the unstable state, $r=0$ and $d r / d t=0$. The accelerated departure from the strong-field area $\left(r \ll r_{o}\right)$ with the growing observable speed $d r / d t=c \sqrt{g_{o o}^{\neq 1}\left(1-g_{o o}^{\neq 1}\right)} \approx c r / r_{o}$ is accompanied by the monotonous decrease of the physical speed $d r / \sqrt{g_{o o}^{\neq 1}} d t=c \sqrt{1-g_{o o}^{\neq 1}} \approx c\left(1-r^{2} / 2 r_{o}^{2}\right)$. Therefore, the acceleration repulsion by strong metric fields diminishes the kinetic energy of accelerated probe bodies (and the measurable temperature of the shared material space). To the contrary, the Newtonian acceleration in weak gravitational fields increases the kinetic energy of probe bodies. The dark energy assumption based on Newtonian reductionism is not working at all for low speed accelerations in strong fields because of the extreme time dilation. The pulsating radial solutions (8) of the dissipation-free equations (7) will gain reshaping of metric densities and their dissipative damping to the static equilibrium, if we take into account neglected radiation exchanges between constant inertial elements of the closed system. 
Nowadays the radial dimension of the Metagalaxy is less than its gravitational scale $R_{o} \equiv G M_{M e t a} / c^{2}$, with $M_{\text {Meta }}=R_{o} c^{2} / G=1.8 \times 10^{53} \mathrm{~kg}$. According to the probe body solution (9) for a constant external field, such dense Metagalaxy should repeal its material elements with acceleration behind the equilibrium proximity $R_{o}(1+\sqrt{2})$. In this way, the Big Bang defragmentation of highly concentrated non-equilibrium matter should start radial accelerations of inertial elements and probe bodies. The dynamical solution of the strong-field limit in (9) provides the acceleration rate $d^{2} R(t) / d t^{2}=c^{2} R(t) / R_{o}^{2} \Rightarrow H_{o}^{2} R(t)$ and the radial speed $d R(t) / d t=H_{o} R(t)$ for further comparisons with the available astronomical data.

Today, Metagalaxy's material continuum is in a very dense phase of expansion with acceleration of the visible monopoles and with cooling of the shared thermal space. After a while, vertexes of the monopoles will continue their radial expansion, but with a slowdown. The self-cooling of the shared space will be monotonous because the Umov'sabsorption of the bi-vertex space energy by the monopole inside heat-energy depends on the monotonous decrease of the physical velocity, not the corresponding initial increase of the visible coordinate speed in ultra strong metric fields. The visible cosmos can reach its lowest space density and temperature in the end of this expansion cycle with monotonous self-cooling. Then such a sparse Metagalaxy will begin a reverse inertial compression with monotonous self-heating. Numerical modeling can provide more detailed information about the global pendulum for 80 billions of galaxies. It would be useful to compare modeling of our cyclic pulsations of dense and sparse shared spaces around the quasi-equilibrium dynamical densities with other oscillating models of the empty space universe, including conformal cyclic 30] and bouncing 31] cosmologies. In general, the dynamics of pulsating thermal systems should take into account the slow exchange of dissipative waves between monopoles through their shared space. This radiation redistributes local kinetic densities and slowly pushes the dynamical continuum of nonlocal thermokinetic energies to the static radial space-matter or a multi-vertex equilibrium state with a crystal symmetry.

\section{Monistic space-matter of kinetic energy with metric stresses instead of gravitation}

\subsection{Second-order redshift to distinguish between curved emptiness and Euclidean space-matter}

The continuous matter-extension with Euclidean 3D geometry and $g_{o o}^{2008}=r^{2} /\left(r+r_{m}\right)^{2}$ in the curved 4-interval predicts [25] the same measurements of Mercury perihelion precession, redshift, and light bending as the Schwrzschild metric with $g_{o o}^{1916}=1-2 r_{m} c^{2} / r$ relative to first-order corrections to the Newton model with weak negative potential $\tilde{\varphi}=-r_{m} / r, r_{m} \equiv G m / c^{2} \ll r$. But the second-order relativistic corrections are different for the empty space gravitation with 1916 Schwarzschild metric [28] and for the 
Euclidean material space of thermokinetic densities with the 2008 metric [19]. Precise measurements of the physical time dilation in the Earth-Sun-Moon system with the time varying weak potential $\tilde{\varphi}$ make it possible to distinguish the 1916 time dilation $(d \tau-d t) / d t \equiv \sqrt{g_{o o}^{1916}}-1 \approx \tilde{\varphi}(1-\tilde{\varphi} / 2)$ in curved empty space from alternative time dilation $\sqrt{g_{o o}^{2008}}-1 \approx \tilde{\varphi}(1+\tilde{\varphi} / 2)$ in the flat material space.

\subsection{D geometry of macroscopic loops in SQUID experiments}

So far, inhomogeneous gravity and arbitrary acceleration never changed SQUID records for long macroscopic contours. Such null results confirm the 3D flatness of the laboratory space with the highest quantum accuracy [32. If someone measures constant gravitational fields or accelerations based on quantization of magnetic flux in microscopic or macroscopic contours, then the Euclidean geometry of the thermal laboratory space and its thermokinetic (pushing) forces will, in principle, be falsified. Supporters of the Schwarzschild metric solution and negative gravitational energies for the physical reality should clearly explain, why all laboratory measurements confirms Euclid line contours with the SQUID accuracy. SQUID accelerometers could be expected only in allegedly warped 3-spaces. Based on the available measurements of quantized flux, one can talk only about a universal (Euclidean) 3-section in the metric dependent 4-interval.

\subsection{ISS can probe local thermokinetic pushes in nonlocal energy organization}

The Dzhanibekov effect, discovered on the geostationary satellite orbit in 1985 and released publicly in 1995, proved that an asymmetric body is prone to periodic redistribution of its kinetic energy over competing degrees of freedom (under a fixed Galois axis) even in the absence of gravity. The geodesic falltakeoff with the constant relativistic energy also maintains periodic pulsations of kinetic energies on inside and outside degrees of freedom. The tendency to extract the inside heat-energy of static inertial elements into the shared material space can be behind their self-assembling in stable symmetrical configurations, including the mono-vertex system and multi-vertex crystals with the constant energy (3). Unexpected aggregation of initially uniform distributions of millimeter-sized particles in the 'informal' discovery of Dr. D.A. Pittit 33] at the International Space Station (ISS) can be explained by the monotonous growth of the shared energy fraction (7) due to the inelastic exchanges to stabilize a new configuration of the diminished monopole energy (5).

Universal self-organizations of multi-vertex systems with almost constant energy (or time-varying system energy due to external EM heat) can be expected in similar experiments on the ISS for millimetersized particles with electric charges [34]. Imaginary charges and real masses have the unified charge-energy 
nature $[21,26]$ in mechanical and electric self-organizations $E_{\text {syst }}=\sum_{k}\left(\sqrt{G} m_{k} \pm i\left|e_{k}\right|\right) \varphi_{G}$, while the electrically charged space is much easier to study for correlated tensions. The hypothesis of 'repulsive attraction' for the dynamical cavity in the cloud of micro-particles [34] can be consistent with balanced thermokinetic stresses in equilibrium material space with steady wave excitations. External accelerating forces in the ISS's and Earth's laboratories impede the study of the electric self-governance through traceable densities of millimeter size in the multi-vertex space-matter of nonlocally distributed energy.

\subsection{Probes of retarded/advanced wave flows and timeless correlations of metric stresses}

The shared inertial space of bi-vertex interference mass-energies (7) can accumulate time-varying elastic and inelastic (wave) extractions from the Umov's inside heat-energy (5) of interacting monopoles. Mechanical waves of inertia (or mechanical photons) have the kinetic (positive, yang) origin. These mechanical photons carry inertial energy-momentum and they can warm the shared continuum (7) for millions of years before absorption by inertial receivers with the back transformation of the wave energy into the inside kinetic capacity (5) of monopoles. If conventional gravitational waves also existed in Newtonian mechanics, they would be unrealistic mathematical solutions with negative (potential, yin) energies. But such negative energies cannot be measured in principle in the physical reality of positive energies. In this way, LIGO measured kinetic waves of pushing inertia of the shared material space with infrequence dispersion, rather than unrealistic waves of negative gravitational energy-potentials.

Almost closed binary systems, such as the Hulse-Taylor binary pulsar PSR 1913+16, slowly extract the inside relativistic heat-energy of the rotating partners into the shared space of interference densities. These monotonous inelastic extractions increase bi-vertex tensions of the material laboratory space. The corresponding 'period decay' of rotating monopoles with diminished energy (5) was explained from the calculated radiation losses [35]. Needless to say for the binary pulsar there is the constancy of its kinetic energy (3) with time-varying mono-vertex and bi-vertex energies (5) and (7), respectively.

Inertial energy integrals of different systems and dissipative wave signals between such systems with independent energy balances cannot move faster than light. The 1st fundamental force arises from the metric stresses of dense (visible) regions under the kinetic organization of one nonlocal system with correlated densities. Such local stresses-pushes are instantaneously agreed over the whole distribution of kinetic densities to hold their energy integral on the constant level. The local stresses within one non-equilibrium distribution are not related to dissipative wave exchanges between different systems or sub-systems, but originate from the elastic cooperation of instantly agreed densities of the nonlocal energyinformation organization. Such local elastic stresses for the 1st force phenomenon of inverse squares do not 
obey the SR rules for the irreversible transport of energy between independent organizations. There are no changes of the Solar system energy (3) under the motion of planets with instant elastic attractions. The timeless stresses and local self-accelerations of correlated densities in non-equilibrium metric organizations explain the Laplace estimations of the Solar system stability for more than few centuries.

All radiation waves can change the local energy density or the temperature of laboratory areas in the shared space-matter with induced stresses. Such time-varying changes of local stresses can be advanced or retarded, but 4-vector accelerations or geodesic force densities keep timeless derivatives in the nonlocal self-organization. The Einstein metric formalism for continuous matter with retarded, timeless, and advanced self-coordination can shed light on the megaparsec correlation of the galaxies [5] and the alignment of the pulsars [4]. And can maintain the Solar activity forecast from the reverse time correlations in the Baikal Deep See Experiment [36.

To accept the Laplace estimations for almost instant forces or our instantaneously agreed metric selfaccelerations in nonlocal energy distribution, one can recalculate directions to the current positions of moving stars and reiterate the corresponding telescopic probes of astrophysicist N. Kozyrev [37. Similar instantaneous correlations of electric charge densities or their macroscopic nonlocality can be tried in electrodynamic probes. The Coulomb's field has already exhibited the rigid motion with timeless 'actionat-a-distance' [38]. To avoid the possible misinterpretation of these well-executed experiments at the Frascati National Laboratory, one more laboratory scheme was proposed [39] with the option to not only verify, but falsify the superluminal exertion of the Coulomb force. Recall that Bohmian mechanics also involves superluminal 'action-at-a-distance' [24]. Nonlocal self-organizations of shared material spaces and instantly correlated densities of multi-vertex macroscopic matter admit both retarded and advanced waves and reversible oscillations [40. Therefore, prospective telescopic and electric experiments should check all three directions for the presence of retarded, instantaneous, and advanced impacts within the nonlocal material organization.

\subsection{To test the monistic structure of metric energy and inertia}

Accurate measurements of the acceleration law (9) for probe bodies in accessible metric fields can reveal a dynamical violation of the inverse squares law for the 1st fundamental force. From the measured accelerations in weak and moderate fields, someone can erroneously assume the inconstancy of the Cavendish parameter, $G(r) \rightarrow G(\infty)\left[1-2 r_{o} / r\right]$, in weak attraction fields. But this is not so because the self-potential $c^{2} / \sqrt{G}$ is to be uniform and universal for all mass-densities and all metric accelerations/decelerations (9). New dynamical probes of the constant G, including cyclotronic tests at different 
altitudes of the laboratory, can verify/falsify the monistic energy structure for physical reality. In monistic mechanics, inertia should universally depend on the variable content of the whole kinetic energy with its metric variations, $m c^{2} \sqrt{g_{o o}^{e x t}} / \sqrt{1-\beta^{2}}$, not the kinetic part $m c^{2} / \sqrt{1-\beta^{2}}$ in dualistic mechanics. According to Newtonian references, gravitational energy does not contribute to inertia of mechanical bodies at all distances from the Earth's centre. Therefore, dual and non-dual approached to inertial energy of macroscopic bodies can be distinguished in practice.

\section{Conclusions}

The principle of equivalence (1) for equilibrium densities and the constant rest-energy integral (3) for the monistic self-organization of topologically different kinetic energies (mono-vertex and bi-vertex) are key points of closed mechanical systems. We used the GR metric formalism to describe Cartesian matterextension with the inside energy variable of mono-vertex densities and the interference energy variable of bi-vertex energies. These complementary variables form local stresses in adaptive inertial distributions with non-equilibrium self-accelerations of metric material densities. Such local self-accelerations simulate the timeless action-at-a-distance between dense regions of the space-matter continuum. The metric selforganization of nonlocal mass-energy should operate only with thermokinetic (measurable) energies that complies with new monistic mechanics, the Yin-Yang dialectics, and Einstein's geometrization of material fields in nondual physics. The negative Poincare pressure, inferred in 1906 to stabilize the Coulomb force in an extended electron, appeared in the monistic mechanics of matter-extension in terms of local kinetic pushes instead of Newtonian pulls by negative (non-existing) energies.

Our two-fraction approach to the locally observable (mono-vertex) and unobservable (bi-vertex) contents of the indivisible reality of monistic (thermokinetic) energy fully supports the Einstein and Infeld directive for the pure field evolution of physics [18]. The metric of continuous kinetic densities becomes not only free from singularities, but also preserves the Euclidean three-dimensional section in the pseudoRiemannian geometry of curved space-time. The continuous inertial charge quantitatively describe all measured GR corrections to Newton's theory [25]. In fact, the Euclidean three-dimensional geometry for thermal physical reality was not directly falsified by measurements such as the verified time dilation (e.g., Gravity Probe A). Continuous energy densities of flat material space in the metric theory of inertia do explain why a well-quantized magnetic flux in superconductors cannot be changed by gravity or accelerations contrary to the predictions of the Schwarzschild [28] metric for empty space. After 1939, Einstein was categorically against the Schwarzschild singularity for the physical reality and this is in favor of our 
developments of General Relativity. Recall that the principled position of 'the reluctant father of black holes' was formulated as a direct response [27] to his opponents (Robertson, Bergman and others) at that time.

Today, one can say that time is not a physical entity, but an abstract concept for describing the retarded/advanced evolution of inertial densities in the physical reality of positive (yang) kinetic energies. Negative (yin) gravitational energies do not belong, in accordance with the Chinese dialectics, the physical reality of the Universe. A metric generalization of Special Relativity for the positive mass-energy at rest cab be focused on the self-organization of only kinetic (yang) energies (3) with competing monovertex and bi-vertex densities. Auxiliary gravitational abstractions with negative energy integrals (4) are insignificant for the self-consistent metric self-governance of positive thermokinetics energies. it is not inertial energies of mono-vertex and bi-vertex elements in Euclidean space, but negative gravitational energies of the curved emptiness with black holes are incorporeal fictions of contemporary pro-Newtonian model.

Be that as it may in theoretical discussions, modern experiments can falsify or support the thermokinetic monism of nonlocal matter with instant interaction lines between dense regions. The nonlocal nature of each inertial system with the shared material space matches the superluminal requirement of Laplace [41] for directions of the 1st fundamental force in the stable Solar system. Newton lured many scientists to the nonexistent world of negative gravitational energies. Nicolas Fatio de Duillier first assumed the anti-Newtonian scheme of pushing gravity in the 1690 letter to Huygens. But minute particles of Fatio and Le Sage (1748) in their kinetic gravitation were even more difficult to accept (due to imminent warming up and speeding down the planets) then the 'divine action-at-a-distance' of Newton. The proposed two-fraction inertia of visible mono-vertex and invisible bi-vertex energies, respectively (5) and (7), admits local pushes of monopoles by the Lomonosov's super-penetrating liquid-matter [42] which strongly resembles the aforementioned bi-vertex fraction of Cartesian space-matter. In this way, the current search for dark energy coincides with the 1742 search for the invisible liquid of Lomonosov for the local reason of accelerations.

Targeted research of inertial matter-extension with the interference kinetic energy (7) and continuously charged matter-extension with the interference electric energy can be commenced in ordinary laboratories. Balanced kinetic energies with the inseparable bi-vertex and mono-vertex fractions can be quantized together in clear terms of positive integrals $m_{k} c^{2}$ within the dynamical ensemble of interacting elements. The nonlocal self-organization and monism of competing kinetic energies (5) and (7) with complementary degrees of freedom and the timeless energy conservation (3) can offer various retarded, advanced, and 
timeless phenomena in physics, chemistry, biology, medicine, informatics and other natural sciences.

Funding: This research received no external funding.

Data Availability Statement: Data available in a publicly accessible repository that does not issue DOIs. The data on kinetic theories of gravitation can be found in the repository Inspire-HEP (inspirehep.net) and in the online libraries en.wikisource.org, gallica.bnf.fr.

Competing interests: The author declare no competing interests.

\section{References}

[1] T S Kuhn The Structure of Scientific Revolutions (Chicago) (1962)

[2] D Lehmkuhl Studies in History and Philosophy of Modern Physics 46316 (2014)

[3] I E Bulyzhenkov Astrophysics and Space Science 363:39 (2018)

[4] D Hutsemékers, L Braibant, V Pelgrims and D Sluse Astronomy and Astrophysics A18 572 (2014)

[5] J H Lee, M Pak, H Song, H-R Lee, S Kim and H Jeong Astrophysical journal 884(2) 104 (2019)

[6] D Garber Descartes' Metaphysical Physics (Chicago: University of Chicago Press)(1992)

[7] D Garber The Cambridge Companion to Descartes (ed) J Cottingham (New York: Cambridge University Press) 286 (1992)

[8] Aristotle Metaphysics (350 B.C.E.) Books 1-14, (trans) W D Ross (The Internet Classics Archive) http://classics.mit.edu//Aristotle/metaphysics.html

[9] A Einstein Ann. Phys. 49 769-822 (1916)

[10] H Poincaré Rendiconti del Circolo Matematico di Palermo 21129 (1906)

[11] I E Bulyzhenkov Physics Letters A 3832367 (2019)

[12] S A Emelyanov Universe 4, 75 (2018)

[13] S Popescu Nature Physics 10264 (2014)

[14] J C Smuts Holism and Evolution, 2nd Edition (London: MacMillian and Co, 2nd Edition) (1927) 
[15] W K Clifford Mathematical Papers (New York, London: MacMillian) 21 (1968, first in 1876)

[16] G Mie Ann. der Physik 37511 (1912), 391 (1912), 401 (1913)

[17] A Einstein, L Infeld and B Hoffmann Annals of Mathematics 3965 (1938)

[18] A Einstein and L Infeld The Evolution of Physics (Cambridge: Cambridge Press)(1938)

[19] I E Bulyzhenkov Int. J. Theor. Phys. 471261 (2008)

[20] I E Bulyzhenkov Jour. Supercond. Nov. Magn. 22723 (2009)

[21] I E Bulyzhenkov Galaxies 6(2) 60 (2019)

[22] N A Umov Schomilch, Zeitschriff d. Math. und Phys. XIX (1874) and Selected works (Moscow:TTL, in Russian) (1950)

[23] L Brillouin Relativity reexamined (New York and London: Academic Press) (1970)

[24] D Bohm Wholeness and the Implicate Order (London: Routledge) (1980)

[25] I E Bulyzhenkov Jour. Mod. Phys. 31465 (2012)

[26] I E Bulyzhenkov Bull. Lebedev Phys. Inst. 43138 (2016)

[27] A Einstein Ann. Math. 40922 (1939)

[28] K Schwarzschild Math. Phys. (Berlin: Sitzungsber. Preuss. Akad. Wiss.) 189 (1916)

[29] J Droste Proc. Kon. Ned Akad. Wet. Amsterdam 25163 (1916-1917). (English translation) Proc. Acad. Sci. Amsterdam 19197 (1917)

[30] R Penrose Cycles of Time: An Extraordinary New View of the Universe (UK:Bodley Head) (2010)

[31] P W Graham, D E Kaplan and S Rajendran Phys. Rev. D97, 044003 (2018)

[32] I E Bulyzhenkov Jour. Supercond. Nov. Magn. 22, 627 (2009)

[33] S G Love, D R Pettit and S R Messenger Meteoritics and Planetary Science 49732 (2014)

[34] M Schwabe et. al. New J. Phys. 19103019 (2017)

[35] J M Weisberg and Y Huang Astrophysical Journal 829 (1) 55 (2016) 
[36] S Korotaev, N Budnev, V Serdyuk, E Kiktenko, D Orekhova and J Gorokhov J. Phys.: Conf. Ser. $1557012026(2020)$

[37] N A Kozyrev Selected publications (Leningrad: Leningrad University Press, in Russian) (1991)

[38] R de Sangro, G Finocchiaro, P Patteri, M Piccolo and G Pizzella The European Physical Journal C. 75137 (2015)

[39] S V Blinov and I E Bulyzhenkov Russian Physics Journal 61321 (2018)

[40] S V Blinov and I E Bulyzhenkov Proceedings of MIPT 8(2) 15 (2016 in Russian)

[41] P S Laplace The System of the World (Sagwan Press) 526 (2018)

[42] M V Lomonosov Complete Works, 11 Vols., Notes on the severity of bodies) 21743 (eds.) S Vavilov and T Kravetz (Moscow and Leningrad: Akad. Nauk. SSSR, in Russian) (1950) 